\title{
On the Impact of Link Scheduling on End-to-End Delays in Large Networks
}

\author{
Jörg Liebeherr, Yashar Ghiassi-Farrokhfal, Almut Burchard
}

\begin{abstract}
We seek to provide an analytical answer whether the impact of link scheduling algorithms on end-to-end delays diminishes on long network paths. The answer is provided through a detailed multi-hop delay analysis, which is applicable to a broad class of scheduling algorithms, and which can account for statistical multiplexing. The analysis is enabled by two contributions: (1) We derive a function that can characterize the available bandwidth at a buffered link for various scheduling algorithms. This characterization is sharp enough to provide necessary and sufficient conditions for satisfying worst-case delay bounds at a single link; (2) We obtain end-to-end delay bounds by solving an optimization problem, in which the service received on a multi-hop path is subsumed into a single function. Since our analysis captures the properties of a broad group of schedulers in a single parameter, it can provide insight how the choice of scheduling algorithms impacts end-to-end delay bounds. An important finding of this paper is that schedulers may exhibit noticeable performance differences which persist in a network setting with long paths.
\end{abstract}

Keywords - Delay Analysis, Scheduling, End-to-End Delays, Network Calculus.

\section{INTRODUCTION}

The importance of link scheduling algorithms at packet switches for trading off assurances on latencies and throughput is well-known, and the differences between link scheduling methods have been studied extensively, e.g., [26]. At the same time, little is known about the role of scheduling algorithms in large networks. The objective of this paper is to shed light on the question whether the impact of link scheduling on end-toend delays diminishes on long network paths.

The literature on the impact of link scheduling on end-to-end performance is limited and not conclusive. Most comparative evaluations of scheduling algorithms investigate only a single link scenario, e.g., [26]. Analytical comparisons between Generalized Processor Sharing (GPS) and Earliest Deadline First (EDF) scheduling over a multi-hop path have been presented for worst-case arrival scenarios [10] and for statistically multiplexed traffic [23], [17]. These works highlight the differences between end-to-end delays with these schedulers, but do not study how the differences evolve as the number of nodes of a network path is increased. Some studies [2], [9], [25] have

Jörg Liebeherr (jorgecomm.utoronto.ca) and Yashar GhiassiFarrokhfal (yashar@comm.utoronto.ca) are with the Department of Electrical and Computer Engineering at the University of Toronto. Almut Burchard (almutamath.utoronto.ca) is with the Department of Mathematics at the University of Toronto. The research in this paper is supported in part by the Natural Sciences and Engineering Research Council of Canada. An earlier version of this paper was presented at the IEEE 30th International Conference on Distributed Computing Systems (ICDCS), Genoa, Italy, June 2010 . found conditions when the output traffic at a link has similar characteristics as the input, suggesting that the end-to-end delay performance of all (work-conserving) scheduling algorithms is similar. An analysis of the throughput performance of FIFO scheduling on long paths in an overloaded network with constant bit rate fluid flow traffic in [11] showed that the end-to-end throughput of a flow asymptotically degrades to that of a lowpriority flow. Our paper is motivated by the question whether a similar degradation is observable under more complex traffic patterns and in networks that are not permanently overloaded.

The analysis in this paper takes a network calculus approach [5], [14], which offers a general method for delay and backlog analysis over a multi-hop path. As a performance metric, we use the end-to-end delay. We assume that the long-term traffic load does not exceed the capacity of any network link. Traffic is characterized in terms of envelope functions and service is characterized by service curve functions. A key result of the (deterministic) network calculus states that the service curve of a multi-hop network path can be computed from the service curves at the links of the path by applying the convolution operator of the min-plus algebra. More precisely, when $S_{1}, S_{2}, \ldots, S_{H}$ are service curves describing the available service for a sequence of links, then the service of the entire network can be expressed by a network service curve $S^{n e t}=S_{1} * S_{2} * \ldots * S_{H}$, where '*' is the min-plus convolution defined in Section II-B. In this fashion, a multi-hop analysis is reduced to the analysis of a single link that represents the entire network path. End-to-end delay bounds computed this way are generally more accurate than adding delay bounds computed for individual links. For example, worstcase delay bounds obtained by adding single-hop delay bounds scale quadratically with the path length, while delays computed with $S^{\text {net }}$ scale linearly [14]. In a probabilistic setting for traffic satisfying exponential bounds, delays computed by adding single-link bounds scale as $O\left(H^{3}\right)$ in the number of traversed links $H$, while delays computed with a network service curve scale with $\Theta(H \log H)$ [4].

In this paper we assess the impact of link scheduling algorithms on probabilistic end-to-end delay bounds using a network calculus analysis. A key difficulty is to find a service curve that can express a probabilistic service guarantee for non-trivial scheduling algorithms. It is possible to express the service available to a traffic flow in terms of the capacity left unused by all other flows with traffic at a buffered link. In a simplified form, 
such a leftover service description is given by:

$$
\text { Leftover service }=\text { Link capacity }-\begin{aligned}
& \text { Arrivals from } \\
& \text { other traffic }
\end{aligned} .
$$

This corresponds to an interpretation of service where the flow under consideration receives a lower priority than all other traffic at the link. For every work-conserving scheduling algorithm (that does not reorder packets from the same flow), such a characterization provides a lower bound for the actually available service. This leftover service interpretation is often referred to as blind multiplexing, since bounds on performance metrics hold even if details of the scheduling algorithm are not available. Refinements of a leftover characterization with service curves that account for specific scheduling algorithms have been considered before [8], [16], [21], however, with the exception of FIFO [8], the accuracy of the characterizations has not been established. An application of the blind multiplexing analysis of [6] to FIFO networks in [12] has concluded that the delay bounds for Markov-modulated arrivals can be very pessimistic.

In this paper, we derive an accurate probabilistic leftover service characterization for a broad class of scheduling algorithms, referred to as $\Delta$-schedulers. The class of $\Delta$-schedulers contains a diverse set of algorithms, such as FIFO, priority scheduling, and EDF. For this class of schedulers, we provide a positive answer to the open question of the achievable accuracy of service curve characterizations, by showing that we can obtain necessary and sufficient conditions for meeting a given deterministic delay bound at a single link.

The tight service curve characterization of schedulers in this paper closes a gap in the literature, with regard to the analysis of end-to-end performance metrics. For a single link, it is feasible to study the relative impact of scheduling and statistical multiplexing on delay performance through a direct analysis of scheduling algorithms, e.g., [3]. However, such a scheduler analysis is not easily extended to multiple hops. Here, the min-plus convolution of service curves in the network calculus offers an elegant alternative for a multi-hop delay analysis. Recent progress on the stochastic network calculus [13] has shown that the convolution of service curves extends to a regime with probabilistic service descriptions. The quality of performance bounds obtained with a network calculus approach critically depends on the accuracy at which scheduling algorithms can be described by service curves. With our tight service curve characterization, we are clearing a path to a more accurate end-toend analysis for the class of $\Delta$-scheduling algorithms.

The probabilistic end-to-end delay bounds derived in this paper extend the stochastic network calculus analysis of [6] from blind multiplexing to all $\Delta$-schedulers. Compared to the blind multiplexing scenario considered in [6], the end-to-end delay analysis of general $\Delta$-schedulers requires an additional analytical optimization (provided in Section IV).

Since our probabilistic analysis contains deterministic delay bounds (that are never violated) as a special case, our work also relates to the literature on worst-case end-to-end analysis [15], [22]. Several methods for computing statistical end-to-end delay bounds have been developed for EDF scheduling under additional assumptions on the operation of the scheduler. Andrews [1] and Li and Knightly [17] consider a so-called coordinated EDF scheduler, where the deadline at downstream links depends on the delay experienced at upstream links. Andrews [1] and Li et. al. [16] assume that traffic exceeding an a priori delay constraint is dropped. Sivaraman and Chiussi [23] present an analysis where traffic is re-shaped at each link, resulting in a non-workconserving system. To the best of our knowledge, the literature does not contain a statistical end-to-end analysis of a network with plain EDF scheduling at each link.

We gain new insights on the role of scheduling algorithms in networks. Even though the asymptotic growth of delays in the path length $H$ for exponentially bounded traffic is $\Theta(H \log H)$ for every $\Delta$-scheduler, we find that, in a non-asymptotic regime, the choice of the scheduling algorithm can have a noticeable impact on end-to-end delays. Thus, accounting for the specifics of a scheduling algorithms can yield probabilistic end-to-end bounds that improve on the conservative bounds provided by blind multiplexing.

The paper is structured as follows. In Section II we discuss the probabilistic characterization of traffic and service in the network calculus, with traffic envelopes and service curves. In Section III we define $\Delta$-scheduling algorithms and analyze their impact on single-hop delays. In Section IV we compute a network service curve for $\Delta$-schedulers and use it for an analytical optimization that result in end-to-end delay bounds. We present numerical examples in Section V and conclude the paper in Section VI.

\section{ARrival And Service Model}

This paper takes a network calculus approach to modeling and analysis, where arrivals of a flow and the service given to a flow are expressed in terms of deterministic or probabilistic bounds. We refer to these bounds as traffic envelopes and service curves, respectively. The concepts presented in this section are developed in [5], [6], [14].

\section{A. Traffic Envelopes}

We consider a continuous-time fluid-flow traffic model where arrivals from a traffic flow or flow aggregate to a service element in the time interval $[0, t)$ are represented by a random process $A(t)$ whose increments satisfy stationary bounds. The model allows instantaneous arrivals of discrete-sized packets, but does not preserve packet boundaries as traffic traverses the network. Traffic departing from a service element in $[0, t)$ is denoted by $D(t)$. Both $A(t)$ and $D(t)$ are nondecreasing in time with $A(t)=D(t)=0$ for $t \leq 0$, and we have $D(t) \leq A(t)$. For brevity, we use the notation $A(s, t)=A(t)-A(s)$ for any $s \leq t$ to denote arrivals in the time interval $[s, t)$. We use a subscript ' $j$ ' to denote traffic arrivals or departures by a flow or flow 
aggregate $j$.

We characterize traffic in terms of traffic envelopes that specify the arrivals over given time intervals. In a worst-case analysis, a deterministic sample path envelope $E$ provides an upper bound on traffic arrivals satisfying for all $t>0$

$$
\sup _{0 \leq s \leq t}\{A(s, t)-E(t-s)\} \leq 0 .
$$

For convenience, we set $E(t)=0$ for $t \leq 0$. By definition, no sample path of $A$ ever violates a deterministic sample path envelope. An example of a deterministic sample path envelope is a leaky bucket with $E(t)=R t+B$ for $t>0$ with a rate parameter $R$ and a burst parameter $B$. Deterministic envelopes are generally conservative bounds, because they do not capture statistical fluctuations of traffic or statistical multiplexing. This motivates the definition of a probabilistic analogue to the deterministic envelope that may be violated with a small probability. A statistical sample path envelope $\mathcal{G}(t)$ with bounding function $\varepsilon(\sigma)$ satisfies for all $t, \sigma \geq 0$

$$
P\left(\sup _{0 \leq s \leq t}\{A(s, t)-\mathcal{G}(t-s)\}>\sigma\right) \leq \varepsilon(\sigma),
$$

where $0 \leq \varepsilon(\sigma) \leq 1$. $\mathcal{G}$ is non-negative with $\mathcal{G}(t)=0$ for $t \leq 0$. The function $\varepsilon(\sigma)$ bounds the probability that a sample path of the arrivals exceeds the envelope $\mathcal{G}$ by more than $\sigma$. Over the years, traffic envelopes have been explored for a wide range of arrival processes, including memoryless, Markov-modulated, and even long range dependent processes. We refer to [19] for an informative survey. For $\varepsilon(\sigma)=0$ if $\sigma>0$, we recover the deterministic version above.

\section{B. Service Curves}

Throughout this paper, we use service curves to describe lower bounds on the service available to a flow. A service element offers a (deterministic) service curve $S$ if the input-output relationship of traffic at the element satisfies for all $t \geq 0$

$$
D(t) \geq \inf _{0 \leq s \leq t}\{A(s)+S(t-s)\} .
$$

The term on the right-hand side is referred to as a min-plus convolution, and denoted by ' $A * S(t)$ '. As examples, a service curve for a constant rate link with capacity $C$ is given by $S(t)=C t$; the service curve for a fixed delay of $d$ is given by $S(t)=\delta_{d}$, where

$$
\delta_{d}(t)= \begin{cases}0, & \text { if } t \leq d \\ \infty, & \text { if } t>d\end{cases}
$$

The service on a path of $H$ service elements, each offering a deterministic service curve of $S_{1}, S_{2}, \ldots, S_{H}$, can be expressed in terms of the convolution $S_{1} * S_{2} * \ldots * S_{H}$. The probabilistic analogue of this concept is a statistical service curve $\mathcal{S}$ with bounding function $\varepsilon(\sigma)$, which satisfies for all $t, \sigma \geq 0$

$$
P\left(D(t)<A *[\mathcal{S}-\sigma]_{+}(t)\right)<\varepsilon(\sigma) .
$$

Here, $\mathcal{S}(t)$ is a non-negative, non-decreasing function with $\mathcal{S}(t)=0$ for $t<0$. Using the notation $[x]_{+}=\max (0, x)$, we denote $[\mathcal{S}-\sigma]_{+}(t)=\max (\mathcal{S}(t)-\sigma, 0)$. The function $\varepsilon(\sigma)$, which bounds the probability that the guarantee of Eq. (3) is violated by more than $\sigma$, is non-increasing and satisfies $0 \leq \varepsilon(\sigma) \leq$ 1 .

When applying the network calculus to practical scheduling methods, one finds that some schedulers are more suitable than others for a service curve description. Some scheduling algorithms, such as General Processor Sharing (GPS) [20] and Service Curve Earliest Deadline (SCED) [8], have been specified in terms of service curves. Generally, however, characterizing a scheduler in terms of service curves requires an indirect description in terms of the available bandwidth capacity that is left unused by other flows with traffic at this link. In the next section, we develop such a description and show that we can completely capture the operation of an entire class of scheduling algorithms.

\section{III. $\Delta$-SCHEDULING AlgoRITHMS}

We consider the arrivals from a set $\mathcal{N}$ of traffic flows (or flow aggregates) to a buffered link with constant rate $C$. The buffer size is assumed large enough so that no losses occur due to buffer overflows. A scheduling algorithm determines the order in which backlogged traffic is transmitted. The buffered link with a scheduling algorithm will be referred to as a node. We assume work-conserving scheduling in the sense that the link transmits at rate $C$ whenever there is backlogged traffic. Scheduling is locally FIFO in the sense that traffic from the same flow is transmitted in the order of its arrival. In this paper, we ignore that packet transmissions cannot be interrupted. This is a reasonable assumption when packet sizes are small compared to the transmission rate. The assumption can be relaxed at the cost of additional notation. We do not assume that traffic shaping is performed between nodes.

Let us consider an arrival from flow $j$ at time $t$, which we refer to as the 'tagged arrival'. This arrival will be transmitted when it has higher precedence than all other backlogged traffic. It turns out that for many scheduling algorithms it is feasible to specify constants $\Delta_{j k}(k \in \mathcal{N})$, so that only arrivals from flow $k$ that occur before $t+\Delta_{j k}$ have higher precedence than the tagged arrival from flow $j$. Note that any locally FIFO $\Delta$-scheduler satisfies $\Delta_{j j}=0$ for all $j \in \mathcal{N}$. We refer to scheduling algorithms whose operations can be completely described by such constants $\Delta_{j k}$ as $\Delta$-schedulers.

Definition 1: Given a set $\mathcal{N}$ of flows with arrivals to a workconserving link. A $\Delta$-scheduler is a work-conserving locally FIFO scheduling algorithm if there exist constants $\left\{\Delta_{j k}\right\}_{j, k \in \mathcal{N}}$ such that an arrival at time $t$ from flow $j$ has precedence precisely over those arrivals from flow $k$ that occur after $t+\Delta_{j k}$.

The class of $\Delta$-schedulers contains a diverse group of scheduling algorithms. 
- FIFO: Traffic is transmitted in the order of arrivals. Consequently, we have $\Delta_{j k}=0$ for all $j, k \in \mathcal{N}$.

- SP, BMUX: With static priority (SP) scheduling, each flow is assigned a priority level, and the scheduler always transmits backlogged traffic with the highest available priority. Traffic within one priority class is served in order of arrival. Since low priority traffic has never higher precedence over higher priority traffic, we have

$$
\Delta_{j k}= \begin{cases}-\infty, & \text { if } k \text { has lower priority than } j, \\ 0, & \text { if } k \text { has the same priority as } j, \\ \infty, & \text { if } k \text { has higher priority than } j .\end{cases}
$$

A scenario where the tagged flow $j$ has low priority and all other traffic has high priority is referred to as blind multiplexing (BMUX). BMUX is an important benchmark, since it yields the highest delays for flow $j$ among all work-conserving locally FIFO schedulers.

- EDF: Each flow $j$ is associated with an a priori delay constraint $d_{j}^{*}$. When traffic from flow $j$ arrives to the scheduler at time $t$, it is assigned a deadline $t+d_{j}^{*}$. Traffic is transmitted in the order of increasing deadlines. Since, in EDF, traffic has a higher precedence if it has a smaller deadline, we have that $\Delta_{j k}=d_{j}^{*}-d_{k}^{*}$ for all $j, k \in \mathcal{N}$. Traffic is transmitted in the order of deadlines, even if an arrival has violated the a priori delay constraint.

There are numerous schedulers that are not $\Delta$-schedulers. Consider GPS [20] as a representative of fair queueing algorithms. Each flow $j$ is assigned a weight $\phi_{j}$ and the amount of service given to backlogged flows is proportional to their weights. Here, at a link with fixed capacity $C$, the service rate of a backlogged flow $j$ at time $t$ is given by $\frac{\phi_{j}}{\sum_{k \in Q(t)} \phi_{k}} C$, where $Q(t)$ is the set of flows that have a backlog at time $t$. Since the set $Q(t)$ is random and the values $\Delta_{j k}$ depend on $Q(t)$, it is not feasible to characterize the $\Delta_{j k}$ by constants. More generally, any scheduling algorithm for which a time limit $\Delta_{j k}$ can only be given in terms of a random variable is not a $\Delta$-scheduler.

\section{A. Service Curves for $\Delta$-Schedulers}

We will characterize the service available to a flow $j \in \mathcal{N}$ at a node with fixed capacity $C$ and a $\Delta$-scheduler in terms of a service curve in the sense of Eq. (5). All flows in $\mathcal{N} \backslash\{j\}$ comprise the cross traffic at the node. Before we present the service curve in Theorem 1 we introduce some notation.

For any locally FIFO scheduler, let $W_{j}(t)$ denote the delay of an arrival from flow $j$ at time $t$, defined as

$$
W_{j}(t)=\inf \left\{s \geq 0 \mid D_{j}(t+s) \geq A_{j}(t)\right\} .
$$

It is convenient to view $W_{j}$ as a function that is defined at all times.

For given constants $\left\{\Delta_{j k}\right\}_{j, k \in \mathcal{N}}$, we define

$$
\Delta_{j k}(y)=\min \left\{\Delta_{j k}, y\right\}
$$

to capture arrivals with higher precedence that occur within a time interval of length $y$. More specifically, consider an arrival from flow $j$ at time $t$ that still resides in the scheduler at time $t+y(y>0)$, i.e., $W_{j}(t)>y$. With respect to this arrival, traffic from flow $k$ that is transmitted earlier must arrive no later than $t+\Delta_{j k}(y)$.

If $\Delta_{j k}=-\infty$, arrivals from flow $k$ never have precedence over traffic from flow $j$, and can be excluded from a delay analysis. We define $\mathcal{N}_{j}$ and $\mathcal{N}_{-j}$ as

$$
\mathcal{N}_{j}=\left\{k \in \mathcal{N} \mid \Delta_{j k}>-\infty\right\}, \quad \mathcal{N}_{-j}=\mathcal{N}_{j} \backslash\{j\},
$$

to specify the subset of flows that may have an impact on the delay $W_{j}$ of traffic from flow $j$.

Finally, we denote by $I($.$) the indicator function, where$ $I($ expr $)=1$ if 'expr' is true, and $I($ expr $)=0$ otherwise. The following theorem specifies a family of statistical service curves for flow $j$ at a node with $\Delta$-scheduling:

Theorem 1: Given a $\Delta$-scheduler operating at a link with capacity $C$ and a set of flows $\mathcal{N}$ with traffic at the link. For each flow $k \in \mathcal{N}$, let $\mathcal{G}_{k}$ be a statistical sample path bound with bounding function $\varepsilon_{k}(\sigma)$ satisfying Eq (2). Then, for each $\theta \geq 0$, the function

$$
\mathcal{S}_{j}(t ; \theta)=\left[C t-\sum_{k \in \mathcal{N}_{-j}} \mathcal{G}_{k}\left(t-\theta+\Delta_{j k}(\theta)\right)\right]_{+} I(t>\theta)
$$

is a statistical service curve with bounding function

$$
\varepsilon_{s}(\sigma)=\inf _{\sum \sigma_{k}=\sigma} \sum_{k \in \mathcal{N}_{-j}} \varepsilon_{k}\left(\sigma_{k}\right) .
$$

The theorem generalizes a service curve characterization that has been known for FIFO [8] to all $\Delta$-schedulers and to a probabilistic setting. In the special case where the arrivals to flow $k$ are bounded by deterministic envelopes $E_{k}$ satisfying Eq. (1), the theorem says that for each $\theta \geq 0$, the function

$$
S_{j}(t ; \theta)=\left[C t-\sum_{k \in \mathcal{N}_{-j}} E_{k}\left(t-\theta+\Delta_{j k}(\theta)\right)\right]_{+} I(t>\theta)
$$

is a deterministic service curve in the sense of Eq. (3).

Proof: Fix a time $t \geq 0$. Let $B_{j k}^{t}(s)$ denote the backlogged traffic from flow $k$ at time $s$ with higher or equal precedence than an arrival from flow $j$ at time $t$. This can be expressed as follows:

$$
\begin{aligned}
B_{j k}^{t}(s) & =A_{k}\left(\min \left\{s, t+\Delta_{j k}\right\}\right)-D_{k}(s) \\
& =A_{k}\left(t+\Delta_{j k}(s-t)\right)-D_{k}(s) .
\end{aligned}
$$

Let $\hat{x}_{t}$ be the last time before $t$ when the scheduler does not have such a backlog, that is,

$$
\hat{x}_{t}=\sup \left\{s \leq t \mid \sum_{k \in \mathcal{N}_{j}} B_{j k}^{t}(s) \leq 0\right\} .
$$

No traffic that arrives earlier than $\hat{x}_{t}$ contributes to the delay of an arrival from flow $j$ at time $t$. Note that Eq. (11) implies that $A_{j}\left(\hat{x}_{t}\right)=D_{j}\left(\hat{x}_{t}\right)$. With Eq. (11), we can rewrite Eq. (6) as

$$
W_{j}(t)=\inf \left\{s \geq 0 \mid D_{j}\left(\hat{x}_{t}, t+s\right) \geq A_{j}\left(\hat{x}_{t}, t\right)\right\} .
$$


Consider first the case where the delay at time $t$ exceeds $\theta$, that is, $W_{j}(t)>\theta$. Since $\Delta$-schedulers are work-conserving, in the entire interval $\left[\hat{x}_{t}, t+\theta\right]$ the link is transmitting traffic from flows in $\mathcal{N}_{j}$ at rate $C$. We get

$$
C\left(\theta+t-\hat{x}_{t}\right)=\sum_{k \in \mathcal{N}_{j}} D_{k}\left(\hat{x}_{t}, t+\theta\right) .
$$

In the interval $\left[\hat{x}_{t}, t+\theta\right]$, the link is only transmitting traffic with higher precedence than the tagged arrival. Since arrivals from flow $k$ with higher precedence than the tagged arrival must occur before $t+\Delta_{j k}(\theta)$, we have for each $k$ that $A_{k}\left(\hat{x}_{t}, t+\Delta_{j k}(\theta)\right) \geq$ $D_{k}\left(\hat{x}_{t}, t+\theta\right)$. Applying this inequality in Eq. (12) gives a lower bound on the transmitted traffic from class $j$ :

$D_{j}\left(\hat{x}_{t}, t+\theta\right) \geq\left[C\left(\theta+t-\hat{x}_{t}\right)-\sum_{k \in \mathcal{N}_{-j}} A_{k}\left(\hat{x}_{t}, t+\Delta_{j k}(\theta)\right)\right]_{+}$.

We can view the right hand term as a lower bound on the capacity available to flow $j$ that is left unused by the other flows. Using that $D_{j}\left(\hat{x}_{t}\right)=A_{j}\left(\hat{x}_{t}\right)$, we can rewrite the inequality as

$$
\begin{aligned}
& D_{j}(t+\theta) \geq A_{j}\left(\hat{x}_{t}\right)+\left[C\left(\theta+t-\hat{x}_{t}\right)\right. \\
& \left.\quad-\sum_{k \in \mathcal{N}_{-j}} A_{k}\left(\hat{x}_{t}, t+\Delta_{j k}(\theta)\right)\right]_{+} I\left(t-\hat{x}_{t}>0\right) .
\end{aligned}
$$

Note that the indicator function always evaluates to 1 . On the other hand, in the case where $W_{j}(t) \leq \theta$, we know that

$$
D_{j}(t+\theta) \geq A_{j}(t),
$$

because the scheduler is locally FIFO.

Eqs. (13) and (14) can be summarized in the statement that

$$
\begin{aligned}
D_{j}(t) & \geq \inf _{s \leq t}\left\{A_{j}(s)+[C(t-s)\right. \\
& \left.\left.-\sum_{k \in \mathcal{N}_{-j}} A_{k}\left(s, t-\theta+\Delta_{j k}(\theta)\right)\right]_{+} I(t-s>\theta)\right\}
\end{aligned}
$$

for all $t \geq 0$ and all $\theta \geq 0$. To see this, we first replace $t$ by $t-\theta$ in both inequalities, then set $s=\hat{x}_{t}$ for Eq. (13) and $s=t-\theta$ for Eq. (14).

Now suppose that we have sample paths so that at time $t$ we have

$$
\begin{aligned}
\sup _{s \leq t}\left\{\sum_{k \in \mathcal{N}_{-j}} A_{k}\left(s, t-\theta+\Delta_{j k}(\theta)\right)\right. \\
\left.\quad-\sum_{k \in \mathcal{N}_{-j}} \mathcal{G}_{k}\left(t-s-\theta+\Delta_{j k}(\theta)\right)-\sigma\right\} \leq 0 .
\end{aligned}
$$

Inserting this into Eq. (15) yields the service curve

$$
\begin{aligned}
D_{j}(t) & \geq \inf _{s \leq t}\left\{A_{j}(s)+[C(t-s)\right. \\
& \left.\left.-\sum_{k \in \mathcal{N}_{-j}} \mathcal{G}_{k}\left(t-s-\theta+\Delta_{j k}(\theta)\right)-\sigma\right]_{+} I(t-s>\theta)\right\} \\
& \geq A_{j} *\left[S_{j}-\sigma\right]_{+}(t ; \theta) .
\end{aligned}
$$

Choosing $\left\{\sigma_{k}\right\}_{k \in \mathcal{N}_{-j}}$ such that $\sigma=\sum_{k \in N_{-j}} \sigma_{k}$, we estimate the bounding function as follows:

$$
\begin{aligned}
& P\left(D_{j}(t) \geq A_{j} *\left[S_{j}-\sigma\right]_{+}(t ; \theta)\right) \\
& \geq P(\text { Eq. (16) holds }) \\
& \geq P\left(\forall k \in \mathcal{N}_{-j}: \sup _{s \leq t}\left\{A_{k}\left(s, t-\theta+\Delta_{j k}(\theta)\right)\right.\right. \\
& \left.\left.\quad-\mathcal{G}_{k}\left(t-s-\theta+\Delta_{j k}(\theta)\right)\right\} \leq \sigma_{k}\right) \quad(18) \\
& \geq 1-\sum_{k \in \mathcal{N}_{-j}} P\left(\operatorname { s u p } _ { s \leq t - \theta + \Delta _ { j k } ( \theta ) } \left\{A_{k}\left(s, t-\theta+\Delta_{j k}(\theta)\right)\right.\right. \\
& \left.\left.\quad-\mathcal{G}_{k}\left(t-\theta+\Delta_{j k}(\theta)-s\right)\right\}>\sigma_{k}\right) \\
& \geq 1-\sum_{k \in \mathcal{N}_{-j}} \varepsilon_{k}\left(\sigma_{k}\right) .
\end{aligned}
$$

In Eq. (18) we restrict the event by requiring that in Eq. (16) each flow $k \in \mathcal{N}_{-j}$ satisfies its envelope $\mathcal{G}_{k}$ for some allowed choice of $\sigma_{k}$. For Eq. (19) we have first applied the union bound and then used that the supremum cannot be assumed for $s>$ $t-\theta+\Delta_{j k}(\theta)$ to restrict the range of $s$. In the last step, we have used that $\mathcal{G}_{k}$ is a statistical sample path bound. The claim follows by minimizing over $\left\{\sigma_{k}\right\}_{k \in \mathcal{N}_{-j}}$.

\section{B. Tightness of the Service Curve}

We make the case that the service curve in Theorem 1 accurately characterizes a scheduler, by showing that we can obtain necessary and sufficient conditions for meeting given delay bounds.

Let $\mathcal{S}_{j}$ be a statistical service curve with bounding function $\varepsilon_{s}(\sigma)$, as defined in Eq. (5), and let $\mathcal{G}_{j}$ be a statistical sample path envelope with bounding function $\varepsilon_{g}(\sigma)$, as defined in Eq. (2). Select $d(\sigma)$ as the smallest value satisfying

$$
\mathcal{G}_{j}(t)+\sigma \leq \mathcal{S}_{j}(t+d(\sigma)), \forall t \geq 0,
$$

and set

$$
\varepsilon(\sigma)=\inf _{\sigma=\sigma_{1}+\sigma_{2}}\left\{\varepsilon_{g}\left(\sigma_{1}\right)+\varepsilon_{s}\left(\sigma_{2}\right)\right\} .
$$

From [6] we have that $d(\sigma)$ is a probabilistic delay bound that satisfies

$$
P\left(W_{j}(t)>d(\sigma)\right)<\varepsilon(\sigma), \forall t \geq 0, \forall \sigma \geq 0 .
$$

We apply this probabilistic delay bound to the service curve of the $\Delta$-scheduler from Theorem 1. Setting $\theta=d(\sigma)$ in Eq. (8), and inserting the service curve into Eq. (20) we obtain

$$
\begin{aligned}
\sup _{t>0}\left\{\mathcal{G}_{j}(t)+\right. & \sigma-[C(t+d(\sigma)) \\
& \left.\left.-\sum_{k \in \mathcal{N}_{-j}} \mathcal{G}_{k}\left(t+\Delta_{j k}(d(\sigma))\right)\right]_{+}\right\} \leq 0 .
\end{aligned}
$$

Since $\Delta_{j j}=0$ in all locally FIFO schedulers, we can replace $\mathcal{G}_{j}(t)$ by $\mathcal{G}_{j}\left(t+\Delta_{j j}(d)\right)$. Collecting terms, we get

$$
\sup _{t>0}\left\{\sum_{k \in \mathcal{N}_{j}} \mathcal{G}_{k}\left(t+\Delta_{j k}(d(\sigma))\right)+\sigma-C t\right\} \leq C d(\sigma) .
$$


For $\sigma=0$ and $d=d(0)$, this condition has the same structure as the probabilistic schedulability conditions from [3], which were derived without using service curves. This indicates that the service curve from Theorem 1 yields similar delay bounds as those obtained with a 'direct' analysis of a specific scheduling algorithm.

The following theorem shows that the deterministic analogue of Eq. (23) is tight in the sense that it yields a necessary and sufficient condition for meeting a delay bound. Here, the envelopes $\mathcal{G}_{k}$ are replaced by deterministic sample-path envelopes $E_{k}$ (satisfying Eq. (1)), $\mathcal{S}_{j}$ is given by Eq. (9), and $\sigma=0, \varepsilon(\sigma)=0$.

Theorem 2: Given a set of flows $\mathcal{N}$ arriving at a buffered link of fixed-rate capacity $C$ and a $\Delta$-scheduler. Assume that the arrivals to each flow $k \in \mathcal{N}$ are bounded by a deterministic envelope function $E_{k}$ satisfying Eq. (1). If

$$
\sup _{t>0}\left\{\sum_{k \in \mathcal{N}_{j}} E_{k}\left(t+\Delta_{j k}(d)\right)-C t\right\} \leq C d
$$

for some $d>0$, then the delay of traffic from flow $j$ does not exceed $d$. If the envelope functions $E_{k}$ are concave, then the condition is tight, in the sense that the worst-case delay is given by $d$.

Condition (24) recovers the necessary and sufficient conditions for meeting a deterministic delay bound $d$ under FIFO, SP, and EDF from [7], [18]. We point out that deterministic envelope functions that provide a tight description for an arrival flow are typically well approximated by concave functions. This follows from the fact that the smallest deterministic envelope function for an arrival sample path is always subadditive.

Proof: Sufficiency of Eq. (24) follows directly from the deterministic version of Eqs. (20) and (22). To prove necessity for concave envelope functions, we assume that the condition in Eq. (24) is violated for $t^{*}$, that is,

$$
\sum_{k \in \mathcal{N}_{j}} E_{k}\left(t^{*}+\Delta_{j k}(d)\right)>C\left(d+t^{*}\right),
$$

and show that this violation results in a delay bound violation.

Consider a transmission scenario where the scheduler is empty at time $t=0$, and immediately after $t=0$, each flow $k \neq j$ generates arrivals to the scheduler such that $A_{k}(t)=$ $E_{k}(t)$. Arrivals from flow $j$ also satisfy $A_{j}(t)=E_{j}(t)$ with the additional requirement that there is an arrival from flow $j$ at $t^{*}$. The backlogged traffic from flow $k$ at time $s$ with higher or equal precedence than an arrival from flow $j$ at time $t^{*}$ is $B_{j k}^{t^{*}}(s)$, as defined in Eq. (10). Defining

$$
B_{j}^{t^{*}}(s)=\sum_{k \in \mathcal{N}_{j}} B_{j k}^{t^{*}}(s),
$$

we obtain that for $s \in\left[0, t^{*}+d\right]$ the following holds:

$$
\begin{aligned}
B_{j}^{t^{*}}(s) & =\sum_{k \in \mathcal{N}_{j}}\left(A_{k}\left(t^{*}+\Delta_{j k}\left(s-t^{*}\right)\right)-D_{k}(s)\right) \\
& =\sum_{k \in \mathcal{N}_{j}} E_{k}\left(t^{*}+\Delta_{j k}\left(s-t^{*}\right)\right)-C s .
\end{aligned}
$$

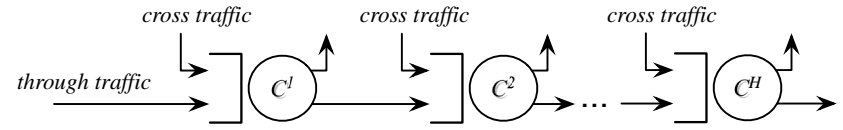

Fig. 1. Multi-hop network.

Since all $E_{k}$ are assumed to be concave and nondecreasing, and $\Delta_{j k}\left(s-t^{*}\right)$ is concave, we have that $B_{j}^{t^{*}}(t)$ is concave in the interval $\left[0, t^{*}+d\right]$. Since $B_{j}^{t^{*}}(0)=0$ by construction of the arrival pattern, and $B_{j}^{t^{*}}\left(t^{*}+\Delta_{j k}(d)\right)>0$ due to the assumption in Eq. (25), we have that $B_{j}^{t^{*}}(s)>0$ for $s \in\left[0, t^{*}+d\right)$. Noting that for $t>t^{*}, B_{j}^{t^{*}}$ describes the backlogged traffic that is transmitted before the arrival from flow $j$ at time $t^{*}$, the arrival cannot be transmitted by time $t^{*}+d$, resulting in a delay bound violation.

\section{END-TO-END DELay ANALYSIS}

We next use the service curve from the previous section to analyze end-to-end delays for $\Delta$-schedulers across a path of multiple hops. We study the delay of a traffic flow (with index ' 0 ') that traverses a sequence of $H$ nodes as shown in Fig. 1, and that experiences cross traffic at each node. We do not assume independence of cross traffic and through traffic. The cross traffic at nodes can be heterogeneous, and we use $\mathcal{N}_{c}^{h}$ to denote the set of cross-traffic flows at the $h$-th node. Each node has a fixed rate capacity, with $C^{h}$ denoting the rate at the $h$-th node. Each node uses a $\Delta$-scheduler for packet transmission, but the scheduling algorithm may be different at each node. We denote by $\Delta_{0 k}^{h}$ the scheduler dependent constants at node $h$ for the through traffic flow 0 and a cross traffic flow $k \in \mathcal{N}_{c}^{h}$. In the derivations we adopt a discrete time system $(t=0,1, \ldots)$.

We compute end-to-end delay bounds for exponentially bounded arrival processes, where the arrivals $A$ of each flow satisfy for all $s \leq t$ and for $\sigma \geq 0$

$$
P(A(s, t)>\rho(t-s)+\sigma) \leq M e^{-\alpha \sigma},
$$

where $M \geq 1$ and $\rho, \alpha>0$ are constants. Such traffic is said to have Exponentially Bounded Burstiness (EBB) [24]. We will write $A \sim(M, \rho, \alpha)$ to denote an EBB arrival process with the given constants.

Remark: For EBB arrivals and a network as in Fig. 1, the asymptotic growth of end-to-end delays in $H$ is $\Theta(H \log H)$ for all $\Delta$-schedulers. This follows from the scaling analysis in [4] for a packetized arrival model. The upper bound of $O(H \log H)$ for delays in BMUX from [4] holds for all $\Delta$-schedulers since delays with BMUX are larger than those of any $\Delta$-scheduler. The lower bound of $\Omega(H \log H)$ in [4] was derived for a network without any cross traffic. Since all $\Delta$-schedulers are locally FIFO, the lower bound holds for any $\Delta$-scheduler.

A statistical sample path envelope satisfying Eq. (2) for the EBB arrival process from Eq. (27) can be computed with the 
union bound as

$$
\mathcal{G}(t)=(\rho+\gamma) t, \quad \varepsilon(\sigma)=\frac{M e^{-\alpha \sigma}}{1-e^{-\alpha \gamma}},
$$

for any choice of $\gamma>0$. We assume that the through traffic is an EBB flow $A_{0} \sim\left(M_{0}, \rho_{0}, \alpha_{0}\right)$ that traverses a path of $H$ nodes. The EBB parameters of each cross traffic flow $k \in \mathcal{N}_{c}^{h}$ is $A_{k} \sim\left(M_{k}, \rho_{k}, \alpha_{k}\right)$. We denote by $\mathcal{G}_{k}$ and $\varepsilon_{k}(\sigma)$, respectively, the statistical sample path envelope and bounding function of flow $k$. Using the statistical sample path envelopes, Theorem 1 provides for the through traffic at the $h$-th node the service curve

$$
\begin{aligned}
& \mathcal{S}^{h}\left(t ; \theta^{h}\right)=\left[C^{h} t\right. \\
& \left.\quad-\sum_{k \in \mathcal{N}_{c}^{h}}\left(\rho_{k}+\gamma\right)\left[t-\theta^{h}+\Delta_{0 k}^{h}\left(\theta^{h}\right)\right]_{+}\right]_{+} I\left(t>\theta^{h}\right), \\
& \varepsilon^{h}(\sigma)=\inf _{\sum_{k \in \mathcal{N}_{c}^{h}} \sigma_{k}=\sigma} \sum_{k \in \mathcal{N}_{c}^{h}} \frac{M_{k} e^{-\alpha_{k} \sigma_{k}}}{1-e^{-\alpha_{k} \gamma}} .
\end{aligned}
$$

We assume that the arrival rates satisfy the stability condition

$$
\rho_{0}+\sum_{k \in \mathcal{N}_{c}^{h}} \rho_{k}<C^{h}, \quad h=1, \ldots, H
$$

and choose the free parameter $\gamma$ to satisfy

$$
0<\gamma<\min _{h} \frac{C^{h}-\sum_{k \in \mathcal{N}_{c}^{h}} \rho_{k}-\rho_{0}}{h+1} .
$$

Since $\gamma$ influences the rate of the envelope as well as the value of the bounding function, the choice of $\gamma$ must make a trade-off. In our analysis, we assume that $\gamma$ is arbitrary, but fixed, and obtain its optimal value numerically.

The value of the violation probability in the above equation can be found by taking advantage of a property derived in [6], which states that, for any set of constants $\left(M_{j}, \rho_{j}, \alpha_{j}\right)$ with $j=$ $1,2, \ldots, N$,

$$
\inf _{\sum_{j=1}^{N} \sigma_{j}=\sigma} \sum_{j=1}^{N} M_{j} e^{-\alpha_{j} \sigma_{j}}=\prod_{j=1}^{N}\left(M_{j} \alpha_{j} w\right)^{\frac{1}{\alpha_{j} w}} e^{-\sigma / w},
$$

where $w=1 / \alpha_{1}+1 / \alpha_{2}+\ldots+1 / \alpha_{N}$. This yields for the bounding function in Eq. (29)

$$
\varepsilon^{h}(\sigma)=M^{h} e^{-\alpha^{h} \sigma},
$$

where

$$
\alpha^{h}=\left(\sum_{k \in \mathcal{N}_{c}^{h}} 1 / \alpha_{k}\right)^{-1}, M^{h}=\prod_{k \in \mathcal{N}_{c}^{h}}\left(\frac{M_{k} \alpha_{k}}{\alpha^{h}\left(1-e^{-\alpha_{k} \gamma}\right)}\right)^{\frac{\alpha^{h}}{\alpha_{k}}} .
$$

The statistical service curve given by Eqs. (28) and (32) describes the service available to the through flow at the $h$-th node. We next use the min-plus algebra of the stochastic network calculus to derive a probabilistic end-to-end delay bound from these per-node service curves.

\section{A. Probabilistic End-to-end Delay Bound}

Following a network calculus approach for computing end-toend delay bounds for a path of nodes, we proceed in two steps. First, we use the per-node service curves for $\Delta$-schedulers above to obtain a statistical network service curve for the through flow on the entire path of $H$ nodes. Second, we use this network service curve in the single-node delay bound from Eq. (20).

Since the bounding function in Eq. (32) has an exponential decay, it clearly satisfies $\int_{0}^{\infty} \varepsilon^{h}(x) d x<\infty$. This allows us to use a result in [6, Theorem 1] which provides a statistical network service curve $\mathcal{S}^{\text {net }}$ satisfying Eq. (5). In the discrete-time version of the network service curve, the through flow receives a service curve for the entire network of $H$ nodes given by

$$
\mathcal{S}^{n e t}(t ; \underline{\theta})=\mathcal{S}^{1} * \mathcal{S}_{\gamma}^{2} * \ldots * \mathcal{S}_{(H-1) \gamma}^{H}(t ; \underline{\theta}),
$$

where $\underline{\theta}=\left(\theta^{1}, \ldots, \theta^{H}\right)$ and $\mathcal{S}_{(h-1) \gamma}^{h}(t ; \theta)=\mathcal{S}^{h}(t ; \theta)-(h-$ 1) $\gamma t$. Compared with the deterministic case, the convolution of statistical service curves incurs at each node an additional rate degradation of $\gamma$. The corresponding bounding function is given by

$$
\varepsilon^{n e t}(\sigma)=\inf _{\sum_{h=1}^{H} \sigma^{h}=\sigma}\left\{\varepsilon^{H}\left(\sigma^{H}\right)+\sum_{h=1}^{H-1} \sum_{j=0}^{\infty} \varepsilon^{h}\left(\sigma^{h}+j \gamma\right)\right\} .
$$

We apply this result to derive $\mathcal{S}^{\text {net }}$ and $\varepsilon^{\text {net }}$ from the per-node service curves given by Eqs. (28) and (32).

For BMUX, that is, $\Delta=\infty$, the functions $\mathcal{S}^{h}$ are simple rate functions and the convolution in (34) is easily computed as the minimum of the rates [6, Eq. (24)]. However, for general $\Delta$, the service curves $\mathcal{S}^{h}$ are neither convex nor concave, and a computation of the convolution in Eq. (34) to obtain $\mathcal{S}^{\text {net }}$ is not straightforward. We resolve this problem by showing that the $\mathcal{S}^{h}$ can be represented as the min-plus convolution of two terms, one of which is concave and the other a simple shift function. With this decomposition and by exploiting basic properties of the min-plus algebra we will be able to obtain an analytical expression for $\mathcal{S}^{\text {net }}$.

By Eq. (30), and since $\theta^{h}-\Delta_{0 k}^{h}\left(\theta^{h}\right)$ is non-decreasing in $\theta^{h}$, the term in the outer brackets of Eq. (28) is non-negative, and we can write

$$
\mathcal{S}_{(h-1) \gamma}^{h}\left(t ; \theta^{h}\right)=\tilde{S}^{h} * \delta_{\theta^{h}}(t) .
$$

Here, $\delta$ is the shift function defined by Eq. (4), and

$$
\begin{aligned}
\tilde{S}^{h}(t)=\left(C^{h}\right. & -(h-1) \gamma)\left(t+\theta^{h}\right) \\
& -\sum_{k \in \mathcal{N}_{c}^{h}}\left(\rho_{k}+\gamma\right)\left[t+\Delta_{0 k}^{h}\left(\theta^{h}\right)\right]_{+} .
\end{aligned}
$$

Note that the term inside the large square brackets of Eq. (28) increases with $\theta^{h}$. We will always take $\theta^{h}$ large enough so that

$$
\left(C^{h}-(h-1) \gamma\right) \theta^{h} \geq \sum_{k \in \mathcal{N}_{c}^{h}}\left(\rho_{k}+\gamma\right)\left[\Delta_{0 k}^{h}\left(\theta^{h}\right)\right]_{+} .
$$


(Otherwise, $\mathcal{S}_{(h-1) \gamma}^{h}$ could be increased by increasing $\theta^{h}$.) With this choice of $\theta^{h}$, we see that $\tilde{S}^{h}$ is nonnegative, strictly increasing, and concave in $t$. Using the associativity and commutativity of the min-plus algebra, we can obtain an expression for $\mathcal{S}^{\text {net }}$ as follows:

$$
\begin{aligned}
\mathcal{S}^{n e t}(t ; \underline{\theta}) & =\left(\tilde{\mathcal{S}}^{1} * \delta_{\theta^{1}}\right) * \ldots *\left(\tilde{\mathcal{S}}_{(H-1) \gamma}^{H} * \delta_{\theta^{H}}\right)(t) \\
& =\left(\tilde{\mathcal{S}}^{1} * \ldots * \tilde{\mathcal{S}}_{(H-1) \gamma}^{H}\right) *\left(\delta_{\theta^{1}} * \ldots * \delta_{\theta^{H}}\right)(t) \\
& =\min _{h=1, \ldots, H}\left\{\tilde{\mathcal{S}}^{h}\right\} * \delta_{\sum_{h=1}^{H} \theta^{h}}(t) \\
& =\min _{h=1, \ldots, H}\left\{\tilde{\mathcal{S}}^{h}\left(t-\sum_{h=1}^{H} \theta^{h}\right)\right\} I\left(t>\sum_{h=1}^{H} \theta^{h}\right) .
\end{aligned}
$$

The second line in the above computation applies the associativity and commutativity of the min-plus convolution. The third line uses that the convolution of concave functions is their pointwise minimum, and that $\delta_{a} * \delta_{b}=\delta_{a+b}$.

For the bounding function $\varepsilon^{\text {net }}$ of the network service curve in Eq. (35) we estimate with Eq. (31) that

$$
\begin{aligned}
\varepsilon^{n e t}(\sigma) & =\inf _{\sum_{h=1}^{H} \sigma^{h}=\sigma}\left\{\frac{M^{H} e^{-\alpha^{H} \sigma^{H}}}{1-e^{-\alpha^{H} \gamma}}+\sum_{h=1}^{H-1} \frac{M^{h} e^{-\alpha^{h} \sigma^{h}}}{\left(1-e^{-\alpha^{h} \gamma}\right)^{2}}\right\} \\
& =M^{n e t} e^{-\alpha^{n e t} \sigma}
\end{aligned}
$$

where

$$
\begin{aligned}
& \alpha^{n e t}=\left(\sum_{h=1}^{H} 1 / \alpha^{h}\right)^{-1}, \\
& M^{n e t} \leq \prod_{h=1}^{H}\left(\frac{M^{h} \alpha^{h}}{\left(1-e^{-\alpha^{h} \gamma}\right)^{2} \alpha^{n e t}}\right)^{\alpha^{n e t} / \alpha^{h}} e^{-\alpha^{n e t} \sigma} .
\end{aligned}
$$

Having derived a network service curve for the through flow in the network shown in Fig. 1, we can now obtain a bound for the end-to-end delays, denoted by $d^{\text {net }}(\sigma)$, by applying the single node delay bound from Eqs. (20)-(22), where $\varepsilon^{\text {net }}$ from Eq. (38) takes the place of $\varepsilon_{s}(\sigma)$, and $\varepsilon_{g}(\sigma)$ is given by $\frac{M_{0} e^{-\alpha_{0} \sigma}}{1-e^{\alpha_{0} \gamma}}$. We evaluate the bounding function in Eq. (22) with another application of Eq. (31) as

$$
\begin{array}{r}
\operatorname{Pr}\left\{W(t) \geq d^{n e t}(\sigma)\right\} \leq\left(\frac{M^{n e t}}{\alpha_{0}}\right)^{\frac{\alpha_{0}}{\alpha_{0}+\alpha^{n e t}}}\left(\frac{M_{0}}{\alpha^{n e t}}\right)^{\frac{\alpha^{n e t}}{\alpha_{0}+\alpha^{n e t}}} \\
\times\left(\alpha^{n e t}+\alpha_{0}\right) e^{\frac{-\alpha_{0} \alpha^{n e t}}{\alpha_{0}+\alpha^{n e t}}} .
\end{array}
$$

It remains to compute $d^{\text {net }}$. Per Eq. (20),

$$
\forall t \geq 0: \mathcal{S}^{n e t}\left(t+d^{n e t}(\sigma) ; \underline{\theta}\right) \geq\left(\rho_{0}+\gamma\right) t+\sigma
$$

is a sufficient condition for $d^{\text {net }}(\sigma)$ to be a probabilistic delay bound. The stability assumption and our choice of $\gamma$ guarantees that Eq. (40) is automatically satisfied for $t$ sufficiently large, since $\mathcal{S}^{\text {net }}(t)$ grows linearly with rate

$$
\min _{h}\left\{C^{h}-h \gamma-\sum_{k \in \mathcal{N}_{c}^{h}} \rho_{k}\right\}>\rho_{0}+\gamma .
$$

Since the left hand side of Eq. (40) is concave in $t$, it suffices to verify the condition in Eq. (40) for $t=0$. Thus, we determine $d^{\text {net }}(\sigma)$ as the smallest $d$ such that

$$
\forall h=1, \ldots, H: \tilde{\mathcal{S}}^{h}\left(d-\sum_{h=1}^{H} \theta^{h}\right) \geq \sigma,
$$

subject to

$$
d^{n e t}(\sigma) \geq \sum_{h=1}^{H} \theta^{h} .
$$

The $\theta^{h}$ 's should be selected to minimize $d^{\text {net }}(\sigma)$, yielding the following optimization problem:

$$
\begin{aligned}
& d^{n e t}(\sigma)=\min _{\theta^{1} \ldots \theta^{h}} \min \left\{d \geq \sum_{h=1}^{H} \theta^{h} \mid\right. \\
& \min _{h=1, \ldots, H}\left\{\left(C^{h}-(h-1) \gamma\right)\left(d+\theta^{h}-\sum_{j=1}^{H} \theta^{j}\right)\right. \\
& \left.\left.-\sum_{k \in \mathcal{N}_{c}^{h}}\left(\rho_{k}+\gamma\right)\left[d+\Delta_{0 k}^{h}\left(\theta^{h}\right)-\sum_{j=1}^{H} \theta^{j}\right]_{+}\right\} \geq \sigma\right\} .
\end{aligned}
$$

Here, the values of $\theta^{h}$ are subject to the constraint in Eq. (37). For $H=1$, since $\theta^{1}-\Delta_{0 k}^{1}\left(\theta^{1}\right)$ is non-decreasing in $\theta^{1}$, the optimal choice is $\theta^{1}=d$ for all $\Delta$-schedulers. This will give the same results as the single node analysis in Section III-B. For $H>1$, the optimal choice of $\theta^{h}$ depends on the value of $\Delta_{0 k}^{h}$.

With the change of variables $X=d^{n e t}(\sigma)-\sum_{h=1}^{H} \theta^{h}$, the optimizing problem takes the following form:

Minimize $d^{n e t}(\sigma)=X+\sum_{h=1}^{H} \theta^{h}$

subject to

$$
\begin{aligned}
& \left(C^{h}-(h-1) \gamma\right)\left(X+\theta^{h}\right)- \\
& \sum_{k \in \mathcal{N}_{c}^{h}}\left(\rho_{k}+\gamma\right)\left[X+\Delta_{0 k}^{h}\left(\theta^{h}\right)\right]_{+} \geq \sigma, \quad h=1, \ldots H, \\
& X \geq 0, \quad \theta^{h} \geq \theta_{*}^{h}, \quad h=1, \ldots H,
\end{aligned}
$$

where $\theta_{*}^{h}$ is the smallest number such that Eq. (37) holds.

Even though this optimization problem is not generally convex, since $\Delta_{0 k}^{h}\left(\theta^{h}\right)$ is concave in $\theta^{h}$ when $\Delta_{0 k}^{h}>0$, it can be solved explicitly as follows. For each value of $X$, let $\theta^{h}(X)$ be the smallest choice of $\theta^{h}$ that satisfies the constraints of the optimization problem. Then $\theta^{h}(X)$ is a piece-wise linear function of $X$, with at most two corners for each element in $\mathcal{N}_{c}^{h}$, where one corner is due to the condition $\theta_{h} \geq \theta_{*}^{h}$, and the other corner is due either to the definition of $\Delta_{0 k}^{h}\left(\theta^{h}\right)$ (if $\Delta_{0 k}^{h}<0$ ) or to enforcing the non-negativity of the term $\left[X+\Delta_{0 k}^{h}\left(\theta^{h}\right)\right]_{+}$(if $\Delta_{0 k}^{h}>0$ ). Thus, $\theta^{h}$ is a piece-wise linear function of $X$, with at most $1+2\left|\mathcal{N}_{c}^{h}\right|$ corners (including $X=0$ ). Inserting these values for $\theta^{h}$, the objective function becomes a piece-wise linear function of the single variable $X$. Since such a function has its minimum at one of the corners, the optimization is solved by evaluating the objective functions for at most $1+\sum_{h=1}^{H}\left(2\left|\mathcal{N}_{c}^{h}\right|\right)$ points, and taking the minimum.

\section{B. Closed-Form Solutions}

We now consider a few illuminating special cases where we can obtain closed-form expressions for the end-to-end delay 
bound. To achieve a closed form we must reduce the set of available parameters. Suppose each node has the same fixed capacity link with rate $C$, and the cross traffic at each node is given by a single flow, given by an identically distributed arrival process $A_{c} \sim\left(M, \rho_{c}, \alpha\right)$. The through flow is given by $A_{0} \sim\left(M, \rho_{0}, \alpha\right)$. We also assume that the same $\Delta$-scheduling algorithm is used at each node, with $\Delta_{0 c}$ denoting the constant for the through traffic flow (with index 0) with respect to the cross traffic flow. With these choices, we obtain $\alpha^{\text {net }}=\alpha / H$, $M^{n e t} \leq \frac{M H}{\left(1-e^{-\alpha \gamma}\right)^{2}}$, and Eq. (39) implies that

$$
\operatorname{Pr}\left\{W(t) \geq d^{n e t}(\sigma)\right\} \leq \frac{M(H+1)}{\left(1-e^{-\alpha \gamma}\right)^{2}} e^{-\frac{\alpha}{H+1} \sigma} .
$$

Since the objective function in Eq. (42) is linear, it assumes its minimum at a point where $H+1$ constraints hold with equality. In such a point, we must have $\theta^{1}=\ldots=\theta^{K}=0$, and $0<$ $\theta^{K+1} \leq \ldots \leq \theta^{H}$ for some $K$. We will now describe how to identify this point.

We reduce the problem to a minimization problem in the single variable $X$. For $X \geq 0$, let $\theta^{h}(X)$ be the smallest nonnegative solution of

$$
(C-(h-1) \gamma)\left(X+\theta^{h}\right)-\left(\rho_{c}+\gamma\right)\left[X+\Delta_{0 c}\left(\theta^{h}\right)\right]_{+} \geq \sigma .
$$

This choice ensures that the $h$-th constraint in Eq. (42) is satisfied with equality whenever $\theta_{h}(X)>0$, and we obtain

$$
d^{n e t}(\sigma)=\min _{X \geq 0}\left\{X+\sum_{h=1}^{H} \theta_{h}(X)\right\} .
$$

Clearly, $\theta^{h}(X)$ is a strictly decreasing function of $X$ in the range where $\theta^{h}(X)>0$. Once we have identified the index $K$ described above, we can drop the first $K$ summands and consider only $\theta^{h}(x)$ for $h>K$. The minimum is assumed at a point where the derivative $\frac{d}{d X}\{$.$\} changes from negative to positive.$

We distinguish two cases. For $\Delta_{0 c} \geq 0$, we compute its derivative as

$$
\frac{d}{d X} \theta^{h}(X)= \begin{cases}-\frac{C-\rho_{c}-h \gamma}{C-(h-1) \gamma}, & \theta^{h}>\Delta_{0 c}, \\ -1, & 0<\theta^{h}<\Delta_{0 c} .\end{cases}
$$

It follows that the optimal $K$ necessarily satisfies

$$
\sum_{h>K} \frac{C-\rho_{c}-h \gamma}{C-(h-1) \gamma}<1
$$

Given $K \geq 1$, we set

$$
X=\frac{\sigma}{C-\rho_{c}-K \gamma} .
$$

For $K=0$ we set $X=0$. The optimal $K$ must also satisfy $\theta^{h}(X)>\Delta_{0 c}$ for all $h>K$. We take $K$ to be the smallest index with these properties. Finally, we determine $d^{\text {net }}(\sigma)$ from Eq. (44).
For $\Delta_{0 c} \leq 0$, the function $\theta^{h}(X)$ is convex, and

$$
\frac{d}{d X} \theta^{h}(X)= \begin{cases}-1, & X<-\Delta_{0 c}, \\ -\frac{C-\rho_{c}-h \gamma}{C-(h-1) \gamma}, & X>-\Delta_{0 c} .\end{cases}
$$

As above, $K$ should be chosen so that Eq. (45) holds. If $K \geq 1$, set

$$
X=\max \left\{\frac{\sigma}{C-(K-1) \gamma}, \frac{\sigma+\left(\rho_{c}+\gamma\right) \Delta_{0 c}}{C-\rho_{c}-K \gamma}\right\} .
$$

If $K=0$ set $X=-\Delta_{0 c}$. We take $K$ to be the smallest index satisfying Eq. (45).

We do not claim that these choices are optimal. However, in practice, $K$ is usually close to $H$, resulting in a near-optimal choice.

To compute a probabilistic delay bound, we first set the right hand side of Eq. (43) equal to the desired violation probability and solve for $\sigma$, and then find $d^{\text {net }}(\sigma)$ by solving the optimization problem in Eq. (42) according to the procedure outlined above. Since there is no explicit term for $\gamma$, the optimization is done numerically over $\gamma$.

There are several special cases where we can find explicit solutions to the optimization problem in Eq. (42):

- $\Delta_{0 c}=\infty$ : This is the case of blind multiplexing. Here, we obtain that the optimal solution is $\theta^{1}=\ldots=\theta^{H}=0$, and we get

$$
d(\sigma)=X=\frac{\sigma}{C-\rho_{c}-H \gamma},
$$

which is the same delay bound found in [6].

- $\Delta_{0 c}=0$ : This is the case of FIFO. Here, the optimization problem greatly simplifies, because the constraints are linear. The second condition in Eq. (45) is always satisfied, leaving only the first condition. We choose $K$ to be the smallest integer that satisfies Eq. (45), determine $X$ from Eq. (46), and compute

$$
\theta^{h}=\frac{(h-K) \gamma X}{C-(h-1) \gamma}, \quad \text { for } h>K .
$$

The resulting delay bound is

$$
d(\sigma)=\frac{\sigma}{C-\rho_{c}-K \gamma}\left(1+\sum_{h>K} \frac{(h-K) \gamma}{C-(h-1) \gamma}\right) .
$$

- $\gamma=0$ : This case arises in a deterministic scenario, in which bounds are never violated. Note that by setting $M=e^{B \alpha}$ and letting $\alpha \rightarrow \infty$ in the EBB model in Eq. (27), we obtain a leaky bucket with $E(t-s)=R(t-s)+B$. From Eq. (42), we see that necessarily $\theta^{h}=\theta$ for all $h$. Then the solution to the optimization gives either $\theta=0$ or $X=0$, resulting in

$$
\begin{aligned}
& d=\min \left\{\frac{\left[B_{0}+H B_{c}-R_{c} \cdot\left[\Delta_{0 c}\right]_{-}\right]_{+}}{C-R_{c}},\right. \\
& \left.\frac{H}{C}\left[\left(B_{0}+H B_{c}+\Delta_{0 c}\right)\right]_{+}\right\} \text {. }
\end{aligned}
$$


For different values of $\Delta_{0 c}$, we obtain end-to-end delay bounds that apply to the deterministic network calculus. For FIFO scheduling, i.e., $\Delta_{0 c}=0$, this is weaker than the corresponding bound obtained in [15], which is given by

$$
d=\frac{B_{0}}{C-R_{c}}+\frac{H}{C} B_{c} .
$$

It remains open whether the difference of the bounds is due to a principal limitation of applying the stochastic network calculus to a deterministic traffic scenario, or if a sharpened analysis of the network service curve may strengthen the result.

Studying the optimization problem, we discover that the delay bounds of FIFO approach that of blind multiplexing when the utilization of the cross traffic $\rho_{c}$ is small or $H$ is large. The reason is that the first condition in Eq. (45) forces $K$ to be close to $H$, which in turn forces Eq. (49) (with $\Delta_{0 c}=0$ ) to converge to the blind multiplexing delay bound Eq. (48). In the numerical examples in the next section, we will see that FIFO approaches the delay bounds of BMUX even for modest path lengths and link utilizations.

\section{NumERICAL EXAMPLES}

We present numerical examples of the end-to-end delay bounds derived in this paper, where we assume that the time unit is $T=1 \mathrm{~ms}$. As traffic, we use discrete-time on-off MarkovModulated Process with two states $(\mathrm{OFF}=1, \mathrm{ON}=2)$, where the probabilities of the transitions $\mathrm{ON} \rightarrow \mathrm{OFF}$ and $\mathrm{OFF} \rightarrow \mathrm{ON}$ are denoted by $p_{12}$ and $p_{21}$. In one time unit in the ON state, the process transmits a fixed amount of data denoted by $P$. We assume that $p_{12}+p_{21} \leq 1$. The effective bandwidth $e b(s, t)=\frac{1}{s t} \log E\left[e^{s A(t)}\right]$ of such a process is bounded by [5]

$$
\begin{aligned}
e b(s, t) \leq \frac{1}{s} \log & \frac{1}{2}\left(p_{11}+p_{22} e^{s P}+\right. \\
& \left.+\sqrt{\left(p_{11}+p_{22} e^{s P}\right)^{2}-4\left(p_{11}+p_{22}-1\right) e^{s P}}\right),
\end{aligned}
$$

where $p_{11}=1-p_{12}$ and $p_{22}=1-p_{21}$. If $A$ denotes the arrivals of an aggregate of $N$ independent such flows, the traffic complies to the EBB model in Eq. (27) with $A \sim(1, N \cdot e b(s, t), s)$.

We use traffic parameters that characterize a compressed video source with high burstiness. The peak rate is set to 1.5 Mbps (megabit per second) and the average rate is $150 \mathrm{kbps}$ (kilobit per second), resulting in a peak-to-average traffic ratio of 10. The average size of a burst, corresponding to a frame, is set to approximately $15 \mathrm{~kb}$. Such a traffic source is modeled by setting the parameters to $P=1.5 \mathrm{~kb}, p_{11}=0.989$ and $p_{22}=0.9$. Both through and cross traffic flows have these characteristics.

We consider a network as in Fig. 1, where all nodes have the same capacity $C=100 \mathrm{Mbps}$, and all nodes use the same $\Delta$ scheduling algorithm, where we set $\Delta_{0 c}:=\Delta$. The number of through flows is $N_{0}$ and the number of cross flows at each node is $N_{c}$. All flows have the same characteristics as discussed

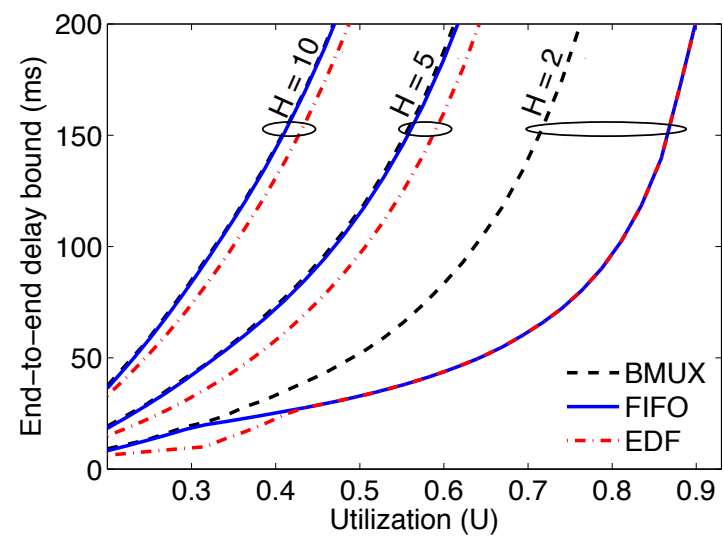

Fig. 2. Example 1: End-to-end delay bounds of through traffic for $\operatorname{EDF}\left(d_{0}^{*}<\right.$ $\left.d_{c}^{*}\right)$, BMUX, and FIFO as a function of the total utilization $U(H=2,5,10$, $U_{0}=15 \%$ (constant), $\varepsilon=10^{-9}$ ).

above. The load on a link is denoted by the utilization $U$, defined as a percentage of utilized capacity $U=\left(N_{0}+N_{c}\right) \cdot 0.15 / 100$. We use $U_{0}$ and $U_{c}$, to denote the utilization due to through and cross traffic, respectively, with $U=U_{0}+U_{c}$. In all examples, we compute end-to-end delay bounds for the through flows with a violation probability set to $\varepsilon=10^{-9}$.

\section{A. Example 1}

We evaluate the end-to-end delay bounds computed in Section IV as a function of the total utilization $U$. We consider the scheduling algorithms BMUX (for reference), FIFO, and EDF. For EDF we set the a priori delay constraints of the through traffic $\left(d_{0}^{*}\right)$ and the cross traffic $\left(d_{c}^{*}\right)$ at each node to satisfy $\Delta=d_{0}^{*}-d_{c}^{*}=-10$. Note that, for scheduling purposes, only the relative difference of the delay constraints is relevant. In the example, the number of through flows is kept constant at $N_{0}=100$, corresponding to a utilization of $U_{o}=15 \%$, while the number of through flows is increased, so that the total utilization $U=U_{0}+U_{c}$ covers a range of $20 \% \leq U \leq 95 \%$. We include results for path lengths of $H=2,5,10$.

In Fig. 2 we see that the delay bounds of FIFO are virtually identical to those of BMUX as early as $H=5$. At the same time, the delay bounds of BMUX and FIFO are noticeably larger than those of EDF, and the difference seems to increase with the network size. On the other hand, for a network with only two hops, $H=2$, the BMUX delays bounds are much higher than those of FIFO and EDF. Note that, since the BMUX bounds from [6] represent the state-of-the-art for end-to-end delay bound analysis, the graphs for EDF illustrate the benefits of applying the scheduler-aware analysis from this paper.

\section{B. Example 2}

This example examines in more detail how scheduling algorithms influence delays in a network. We use the same setup as in Example 1, however, we keep the link utilization constant at $U=50 \%$ and instead vary the traffic mix $U_{c} / U$. For EDF we consider two cases: shorter delay constraints for through traf- 


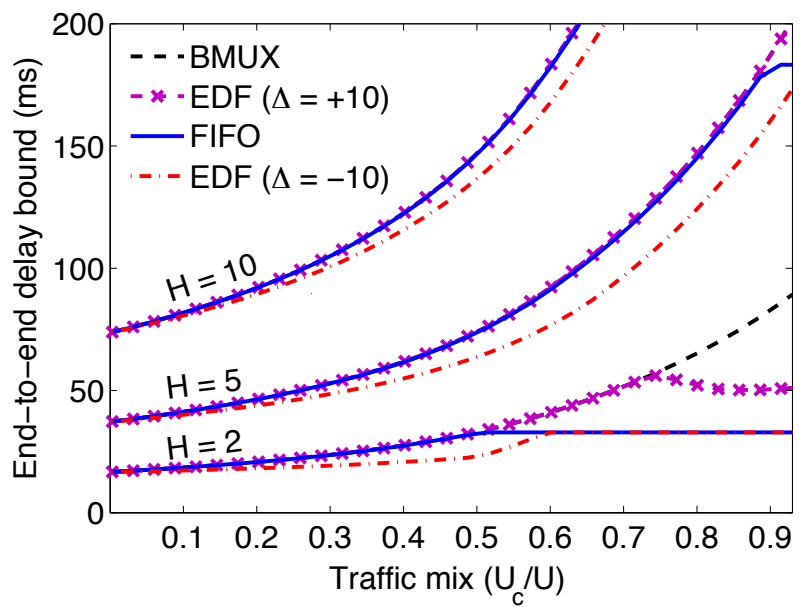

Fig. 3. Example 2: End-to-end delay bounds of through traffic as a function of the traffic mix $U_{c} / U\left(H=2,5,10, U=50 \%\right.$ (constant), $\left.\varepsilon=10^{-9}\right)$.

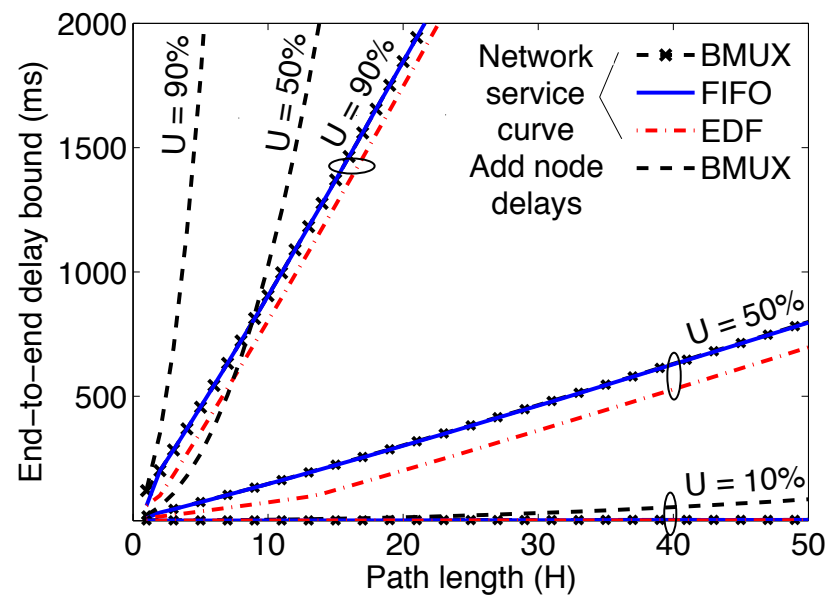

Fig. 4. Example 3: End-to-end delay bounds of through traffic vs. path length $\left(U_{0}=U_{c}, U=10,50,90 \%, \varepsilon=10^{-9}\right)$.

fic $(\Delta=-10)$ and longer delay constraints for through traffic $(\Delta=10)$. The results are shown in Fig. 2. Even though the total utilization at the node is constant, we observe different delay bounds for the evaluated schedulers. Interpreting FIFO as an EDF scheduler with identical a priori delay constraints, i.e., $d_{0}^{*}=d_{c}^{*}$, and BMUX as an EDF scheduler with $d_{0}^{*}=\infty$ and $d_{c}^{*}<\infty$, we see, for $H=2$, that for $d_{0}^{*}>d_{c}^{*}$ end-to-end delay bounds become more sensitive to increased cross traffic. On the other hand, for $d_{0}^{*}<d_{c}^{*}$, we see less sensitivity to the traffic mix. This is expected, since cross traffic mostly has lower precedence, while through traffic maintains its locally FIFO property. When the path length $H$ is increased, these effects are diminished as the scaling law for the delays of $\Delta$-schedulers makes all schedulers have a similar performance as BMUX, where increased cross traffic results in a steep increase of delay bounds.

\section{Example 3}

We illustrate the scaling of end-to-end delay bounds as a function of the path length $H$. Using the same MMPP traffic parameters as before, we set $N_{0}=N_{c}$, and compute the delay

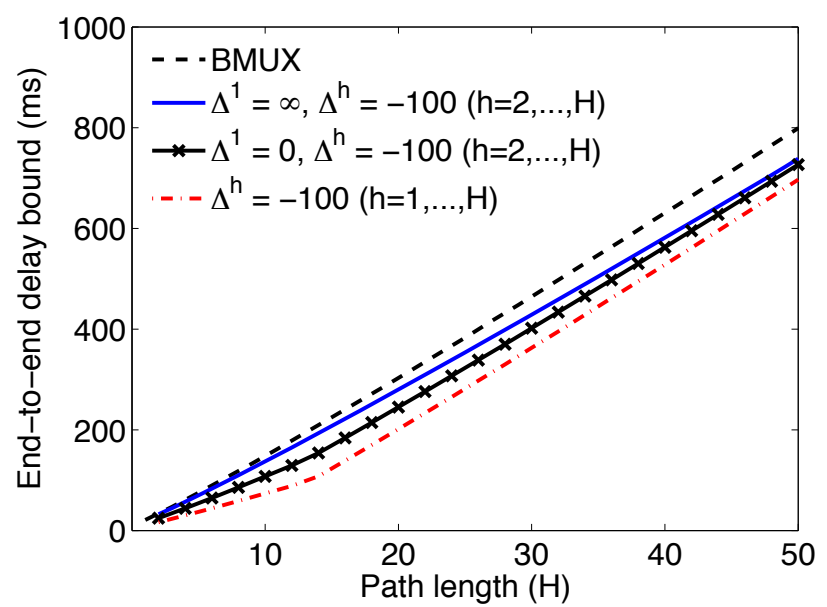

Fig. 5. Example 4: End-to-end delay bounds with mix of link schedulers $\left(U_{0}=U_{c}, U=50 \%, \varepsilon=10^{-9}\right)$.

bounds for different values of $U=10,50,90 \%$. For EDF, we set delay constraints at each node so that $\Delta=-100$, given a shorter a priori delay constraint to the cross traffic. Since we are considering longer path lengths, which incur longer delays, we have changed the value of $\Delta$ compared to the previous examples. For BMUX, FIFO, and EDF we compute delay bounds as discussed in Section IV. For BMUX, we additionally present a delay analysis that is based on adding up per-node delay bounds, using a discrete-time version of such an analysis in $[6, \mathrm{Sec}-$ tion IV.A]. In Fig. 4 we see that the method of adding per-node delay bounds yields loose bounds. In fact, the growth of delays can be shown to be $O\left(H^{3} \log H\right)$ with discrete time. The delay bounds using our network service curve increase essentially linearly for all schedulers, following the predicted asymptotic growth of $\Theta(H \log H)$. These findings generalize a comparison of a network delay analysis with a node-by-node analysis for BMUX in [6] for blind multiplexing. Note that, for the depicted range of $H$, the delay bounds for FIFO and BMUX appear identical when network nodes have a higher load, while delays for EDF (with the selected a priori delay constraints) are noticeably lower.

\section{Example 4}

Taking advantage of our ability to analyze heterogeneous networks, we evaluate the impact of changing the link scheduling at a single node on a network path with $H$ nodes. We use the same traffic characteristics as before, with $U=50 \%$ and $N_{0}=N_{c}$. The results, shown in Fig. 5, consider several scenarios. BMUX indicates a network where all nodes give lower priority to the through traffic, i.e., $\Delta^{h}=\infty$. As expected this results in the largest delays for the through traffic. The curve that yields the lowest delays is labeled as ' $\Delta^{h}=-100(h=1, \ldots, H)$ '. This is a scenario where all schedulers are EDF, and where the through traffic has a delay constraint which is $100 \mathrm{~ms}$ shorter than that of the cross traffic. The remaining curves use a mix of schedulers. In the curve labeled ' $\Delta^{1}=0, \Delta^{h}=-100(h=$ 
$2, \ldots, H)^{\prime}$, the first node uses FIFO and the other nodes use EDF with the given delay constraints. And the curve labeled ' $\Delta^{1}=\infty, \Delta^{h}=-100(h=2, \ldots, H)$ ', has BMUX at the first node. The results indicate that changing a single scheduler on a path can have a noticeable impact on the end-to-end delays. While the distortion caused by the 'different' scheduler appears to gradually increase with large $H$, even for $H=50$ the impact of changing a single scheduler on this path remains noticeable.

\section{CONCLUSION}

We presented an end-to-end analysis of probabilistic delay bounds for $\Delta$-schedulers, a class of algorithms that can be defined in terms of constants which specify time intervals when arrivals have precedence over buffered traffic. We derived a statistical service curve that can characterize the operations of any given $\Delta$-scheduler to a degree that it recovers tight delay bound conditions. We performed an end-to-end delay bound analysis by providing explicit solutions to an optimization problem. We presented numerical examples that illustrated the impact of the choice of the scheduling algorithm on end-to-end delays. We observed that delay bounds of FIFO frequently approach those of blind multiplexing when the number of traversed nodes is large. At the same time, the delays of EDF scheduling were generally markedly different, and maintained the difference on long paths. Thus, our results suggest that an accurate end-toend analysis should take into consideration the specifics of the scheduling algorithms at packet switches.

\section{REFERENCES}

[1] M. Andrews. Probabilistic end-to-end delay bounds for earliest deadline first scheduling. In Proc. IEEE Infocom, pages 603-612, March 2000.

[2] T. Bonald, A. Proutiere, and J. W. Roberts. Statistical guarantees for statistical guarantees for streaming flows using expedited forwarding. In Proc. IEEE Infocom, pages 1104-1112, April 2001.

[3] R. Boorstyn, A. Burchard, J. Liebeherr, and C. Oottamakorn. Statistical service assurances for traffic scheduling algorithms. IEEE Journal on Selected Areas in Communications, 18(12):2651-2664, December 2000.

[4] A. Burchard, J. Liebeherr, and F. Ciucu. On $\Theta(H \log H)$ scaling of network delays. In Proc. of IEEE Infocom, pages 1866-1874, May 2007.

[5] C.-S. Chang. Performance Guarantees in Communication Networks. Springer Verlag, 2000.

[6] F. Ciucu, A. Burchard, and J. Liebeherr. Scaling properties of statistical end-to-end bounds in the network calculus. IEEE Transactions on Information Theory, 52(6):2300-2312, June 2006.

[7] R. Cruz. A calculus for network delay, parts I and II. IEEE Transactions on Information Theory, 37(1):114-141, January 1991.

[8] R. L. Cruz. SCED+: Efficient management of quality of service guarantees. In Proc. IEEE Infocom, pages 625-634, March 1998.

[9] D. Y. Eun and N. B. Shroff. Network decomposition: Theory and practice, to appear in IEEE/ACM Trans. on Networking, 13(3):526-539, June 2005.

[10] L. Georgiadis, R. Guerin, V. Peris, and K. Sivarajan. Efficient network QoS provisioning based on per node traffic shaping. IEEE/ACM Transactions on Networking, 4(4):482-501, Aug. 1996.

[11] Y. Ghiassi-Farrokhfal and J. Liebeherr. Output characterization of constant bit rate traffic in fifo networks. IEEE Communications Letters, 13(8):618-620, August 2009.

[12] P. Giacomazzi. Closed-form analysis of end-toend network delay with markov-modulated poission and fluid traffic. Computer Communications, 32(4):640-648, March 2009.

[13] Y. Jiang and Y. Liu. Stochastic Network Calculus. Springer, 2008.
[14] J. Y. Le Boudec and P. Thiran. Network Calculus. Springer Verlag, Lecture Notes in Computer Science, LNCS 2050, 2001.

[15] L. Lenzini, E. Mingozzi, and G. Stea. Delay bounds for fifo aggregates: A case study. Computer Communications, 28(3), 2005.

[16] C. Li, A. Burchard, and J. Liebeherr. A network calculus with effective bandwidth. IEEE/ACM Trans. on Networking, 15(6):1442-1453, December 2007.

[17] C. Li and E. W. Knightly. Coordinated multihop scheduling: A framework for end-to-end services. IEEE/ACM Transactions on Networking, 10(6):776-789, December 2002.

[18] J. Liebeherr, D. Wrege, and D. Ferrari. Exact admission control for networks with bounded delay services. IEEE/ACM Transactions on Networking, 4(6):885-901, December 1996.

[19] S. Mao and S. S. Panwar. A survey of envelope processes and their applications in quality of service provisioning. IEEE Communications Surveys \& Tutorials, 8(3):2-20, $3^{\text {rd }}$ Quarter 2006.

[20] A. K. Parekh and R. G. Gallager. A Generalized Processor Sharing Approach to Flow Control in Integrated Services Networks: the Single-Node Case. IEEE/ACM Transactions on Networking, 1(3):344-357, June 1993.

[21] J. Qiu and E. W. Knightly. Inter-class resource sharing using statistical service envelopes. In Proc. IEEE Infocom, pages 1404-1411, March 1999.

[22] J. B. Schmitt, F. A. Zdarsky, and M. Fidler. Delay bounds under arbitrary multiplexing: When network calculus leaves you in the lurch. In Proc. IEEE Infocom, pages 1669-1677, April 2008.

[23] V. Sivaraman and F. M. Chiussi. Providing end-to-end statistical delay guarantees with earliest deadline first scheduling and per-hop traffic shaping. In Proc. IEEE Infocom, pages 603-612, March 2000.

[24] O. Yaron and M. Sidi. Performance and stability of communication networks via robust exponential bounds. IEEE/ACM Transactions on Networking, 1(3):372-385, June 1993.

[25] Y. Ying, R. Mazumdar, C. Rosenberg, and F. Guillemin. The burstiness behavior of regulated flows in networks. In Proc. 4th IFIP Networking 2005, pages 918-929, May 2005.

[26] H. Zhang. Service disciplines for guaranteed performance service in packet-switching networks. Proceedings of the IEEE, 83(10):1374-1396, Oct. 1995

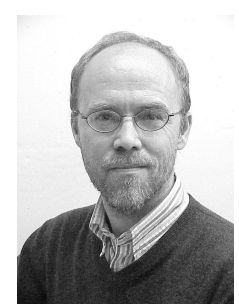

Jörg Liebeherr (S'88, M'92, SM'03, F'08) received the $\mathrm{Ph} . \mathrm{D}$. degree in Computer Science from the Georgia Institute of Technology in 1991. He was on the faculty of the Department of Computer Science at the University of Virginia from 1992-2005. Since Fall 2005, he is with the University of Toronto as Professor of Electrical and Computer Engineering and Nortel Chair of Network Architecture and Services.

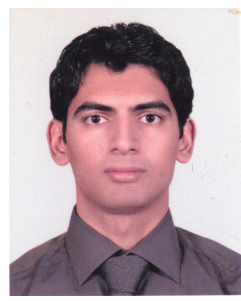

Yashar Ghiassi-Farrokhfal (S'05) holds an B.S. degree from Amirkabir University of Technology in 2003, and an M.S. degree from Sharif University of Technology in 2005, both in Electrical Engineering. $\mathrm{He}$ is currently a Ph.D. student in the Department of Electrical and Computer Engineering at the University of Toronto.

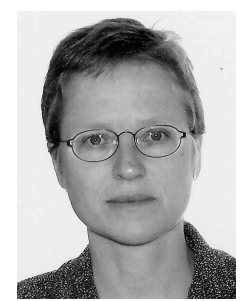

Almut Burchard received the Ph.D. degree in Mathematics from the Georgia Institute of Technology in 1994. She was on the faculty of the Department of Mathematics at Princeton University (1994-1998) and the University of Virginia (1998-2005). She is currently a Professor of Mathematics at the University of Toronto. 